and Zanovello, P. 2003. L-arginine metabolism in myeloid cells controls T-lymphocyte functions. Trends Immunol. 24:302-306.

14. Rodriguez, P.C., et al. 2004. Arginase I production in the tumor microenvironment by mature myeloid cells inhibits T-cell receptor expression and antigen-specific T-cell responses. Cancer Res. 64:5839-5849.

15. Koneru, M., Schaer, D., Monu, N., Ayala, A., and Frey, A.B. 2005. Defective proximal TCR signaling inhibits CD8+ tumor-infiltrating lymphocyte lytic function. J. Immunol. 174:1830-1840.

16. Franco, J.L., et al. 1995. Partial degradation of T-cell signal transduction molecules by contaminating granulocytes during protein extraction of splenic $\mathrm{T}$ cells from tumor-bearing mice. Cancer Res. 55:3840-3846.

17. Levey, D., and Srivastava, P. 1995. T cells from late tumor-bearing mice express normal levels of p56lck, p59fyn, ZAP-70 and CD3zeta despite suppressed cytolytic activity. J. Exp. Med. 182:1029-1036.

18. Terabe, M., et al. 2003. Transforming growth factor-beta production and myeloid cells are an effector mechanism through which CD1d-restricted T cells block cytotoxic T lymphocyte-mediated tumor immunosurveillance: abrogation prevents tumor recurrence. J. Exp. Med. 198:1741-1752.

19. Hansen, D.S., Siomos, M.A., Buckingham, L., Scalzo, A.A., and Schofield, L. 2003. Regulation of murine cerebral malaria pathogenesis by CD1drestricted NKT cells and the natural killer complex. Immunity. 18:391-402.

20. Terabe, M., et al. 2000. NKT cell-mediated repression of tumor immunosurveillance by IL-13 and the IL-4R-STAT6 pathway. Nat. Immunol. 1:515-520.

21. Gordon, S. 2003. Alternative activation of macrophages. Nat. Rev. Immunol. 3:23-35

22. Mantovani, A., Sozzani, S., Locati, M., Allavena, P., and Sica, A. 2002. Macrophage polarization: tumor-associated macrophages as a paradigm for polarized M2 mononuclear phagocytes. Trends Immunol. 23:549-555.

23. Sinha, P., Clements, V.K., and Ostrand-Rosenberg, S. 2005. Reduction of myeloid-derived suppressor cells and induction of M1 macrophages facilitate the rejection of established metastatic disease. J. Immunol. 174:636-645.

24. Xia, Y., Roman, L.J., Masters, B.S., and Zweier, J.L. 1998. Inducible nitric-oxide synthase generates superoxide from the reductase domain. J. Biol.
Chem. 273:22635-22639.

25. Brito, C., et al. 1999. Peroxynitrite inhibits T lymphocyte activation and proliferation by promoting impairment of tyrosine phosphorylation and peroxynitrite-driven apoptotic death. J. Immunol. 162:3356-3366

26. De Santo, C., et al. 2005. Nitroaspirin corrects immune dysfunction in tumor-bearing hosts and promotes tumor eradication by cancer vaccination. Proc. Natl. Acad. Sci. U. S. A. 102:4185-4190.

27. Rodriguez, P.C., et al. 2005. Arginase I in myeloid suppressor cells is induced by COX-2 in lung carcinoma. J. Exp. Med. 202:931-939.

28. Nefedova, Y., et al. 2004. Hyperactivation of STAT3 is involved in abnormal differentiation of dendritic cells in cancer. J. Immunol. 172:464-474.

29. Gabrilovich, D. 2004. Mechanisms and functional significance of tumour-induced dendritic-cell defects. Nat. Rev. Immunol. 4:941-952.

30. Enk, A.H., Jonuleit, H., Saloga, J., and Knop, J. 1997. Dendritic cells as mediators of tumor-induced tolerance in metastatic melanoma. Int. J. Cancer. 73:309-316.

31. Reis e Sousa, C. 2006. Dendritic cells in a mature age. Nat. Rev. Immunol. 6:476-483.

\title{
To be or not to be B7
}

\author{
Xingxing Zang and James P. Allison
}

Immunology Program, Howard Hughes Medical Institute, Memorial Sloan-Kettering Cancer Center, New York, New York, USA.

\begin{abstract}
The activation of lymphocytes and development of adaptive immune responses is initiated by the engagement of TCRs by antigenic peptide-MHC complexes and shaped at the clonal level by both positive and negative costimulatory signals. The $\mathrm{B} 7$ family members are involved at several stages in this process. In this issue of the JCI, Vogt et al. show that the B7 family-related protein V-set and Ig domain-containing 4 (VSIG4) can act as an inhibitor of $T$ cell activation (see the related article beginning on page 2817). Intriguingly, the same molecule was recently independently identified as a complement receptor of the Ig superfamily (CRIg) and was convincingly demonstrated to be a receptor for complement component 3 fragments. These findings raise interesting questions regarding the physiological roles and mechanisms of action of this molecule. Identification of dual functions of this molecule provides an additional level of complexity in $\mathrm{T}$ cell costimulation.
\end{abstract}

Members of the B7 family provide crucial costimulatory signals that regulate $\mathrm{T}$ cell responses. The major role of the classic costimulatory molecules B7-1, B7-2/CD28, and CTL antigen-4 (CTLA-4) is to regulate $\mathrm{T}$ cell responses at an early stage of $\mathrm{T}$ cell activation in lymphoid tissues. In the past few years,

Nonstandard abbreviations used: C3, complement component 3; CR1, complement receptor 1; CRIg, complement receptor of the Ig superfamily; CTLA-4, CTL antigen-4; PDCD1, programmed cell death 1; VSIG4, $\mathrm{V}$-set and Ig domain-containing 4.

Conflict of interest: The authors have declared that no conflict of interest exists.

Citation for this article: J. Clin. Invest. 116:2590-2593 (2006). doi:10.1172/JCI30103. several new members of the extended B7/CD28 family have been discovered. These include: (a) ICOS ligand (ICOSL; also known as B7h, ICOS-L, B7RP-1, B7-H2) and its receptor ICOS (also known as AILIM, CD278); (b) CD274 (also known as PD-L1, B7-H, B7-H1) and programmed cell death 1 ligand 2 (PDCD1LG2; also known as PD-L2, B7-H2, B7-DC) and their counterreceptor programmed cell death 1 (PDCD1; also known as PD-1); and (c) CD276 (also known as B7-H3, B7RP-2) and $\mathrm{V}$-set domain-containing $\mathrm{T}$ cell activation inhibitor 1 (VTCN1; also known as B7-H4, B7X, B7S1), whose counterreceptors remain unidentified (1). Their precise roles in $\mathrm{T}$ cell regulation are just now being elucidated, but it is apparent that these molecules play important roles in regulating cell-mediated immune responses both temporally and spatially. The blockade of the inhibitory effects of CTLA-4 on T cell proliferation using monoclonal antibodies has been shown to be quite powerful as a means of enhancing immune responses to tumors in mouse studies, and significant clinical effects have been observed in trials of anti-CTLA-4 in melanoma, renal, ovarian, and prostate cancer (2). Similarly, blockade of PDCD1 has been shown to restore the function of exhausted $\mathrm{CD}^{+}$ $\mathrm{T}$ cells during infection and accelerate tumor eradication $(3,4)$. Because of their fundamental biological importance and therapeutic potential, there has been considerable interest in the identification of molecules with costimulatory activity. In this issue of the JCI, Vogt et al. (5) describe a B7 family-related molecule, V-set and Ig domain-containing 4 (VSIG4, also known as Z39Ig [ref. 6] and complement receptor of the Ig superfamily (CRIg) [ref. 7]) and show that it can function as an inhibitor of $\mathrm{T}$ cell activation. Interestingly, a prior study had shown that this molecule was also a complement receptor on macrophages and is required for phagocytosis of circulating pathogens (7). 
A

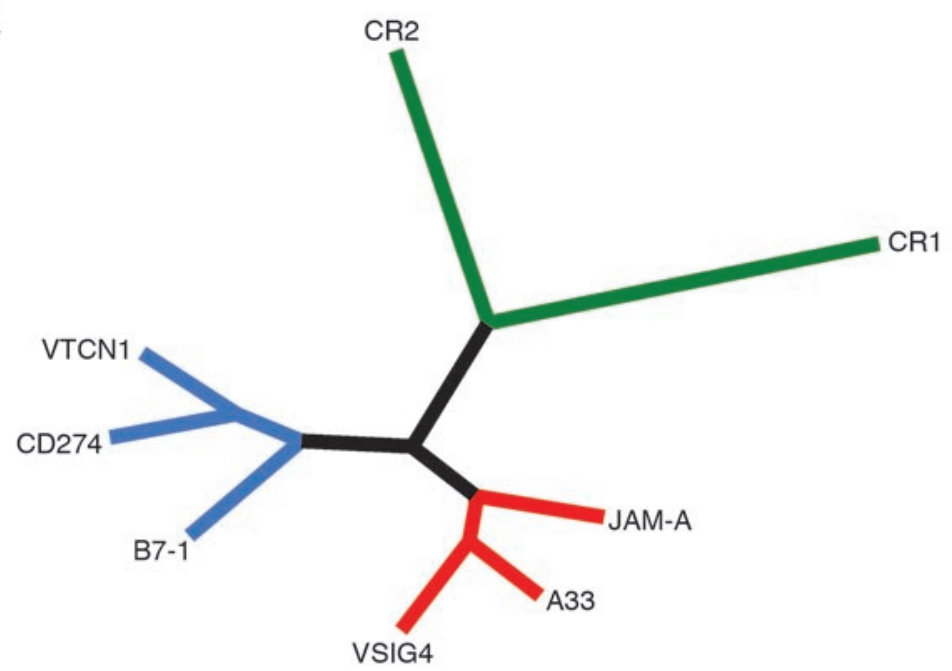

B

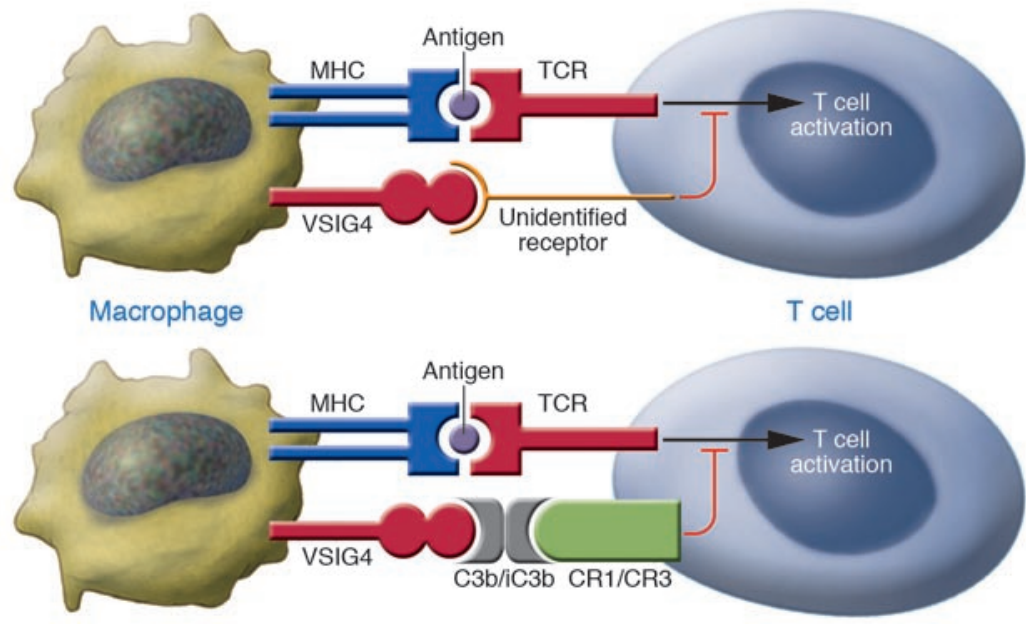

\section{Figure 1}

Phylogeny of VSIG4 and hypothetical models of its role as a T cell inhibitor. (A) VSIG4, A33, and junctional adhesion molecule A (JAM-A) are grouped together in a phylogenetic tree, whereas representatives of the B7 family (B7-1, CD274, and V-set domain-containing $T$ cell activation inhibitor 1 [VTCN1]) and CR1 and $\mathrm{CR} 2$ are divergent. All the sequence alignments and homology comparisons were performed with MacVector 7.0. (Accelrys). The phylogenetic tree was generated by Phylogenetic Analysis Using Parsimony (PAUP 4.0b10; Sinauer Associates Inc.) with sequence alignment by removal of significant inserts and trimming $\mathrm{C}$ - and $\mathrm{N}$-terminal extensions. (B) T cells express an unidentified receptor for VSIG4 expressed on macrophages. The ligation of this receptor downregulates TCR-mediated signaling to the nucleus. Alternatively, VSIG4 on macrophages and CR1/CR3 on T cells bind the same multimeric C3b or iC3b molecules, which in turn triggers a signal resulting in inhibition of $\mathrm{T}$ cell activation.

\section{Z39lg, CRIg, and VSIG4: expression and evolution}

VSIG4 was initially cloned as an Ig superfamily gene (Z39Ig) located at the pericentromeric region of the human $\mathrm{X}$ chromosome (6). Z39IG RNA message is highly expressed in murine liver and heart and in human lung, placenta, adrenal gland, and heart $(5,7)$. Monoclonal antibodies against VSIG4 have recently been generated and have revealed that VSIG4 is expressed by monocyte-derived macrophages $(7,8)$. Interestingly, VSIG4 is mainly expressed on resident macrophages in the liver, heart, adrenal gland, lung, and peritoneal cavity. However, only a subset of tissue-resident macrophages expresses VSIG4, and the phenotypic and functional differences between VSIG4-positive and -negative tissue macrophages are not clear. Unlike conventional B7 family members, VSIG4 is constitutively internalized from the cell surface and is located in a pool of recycling membranes
(7). This process occurs in a ligand-independent fashion. In the presence of ligands, VSIG4 returns to the recycling compartment prior to phagosome-lysosome fusion, thus avoiding degradation.

Like many members of the Ig superfamily, VSIG4 has extracellular IgV and IgC domains; this structure is conserved in most mammalian species. However, the mouse VSIG4 as well as a human spliced form lack an extracellular IgC domain (5, 7). Given that both human VSIG4 and mouse VSIG4 can bind the ligands, the extracellular $\operatorname{IgV}$ must be the functional domain. An interesting phylogenetic relationship between VSIG4 and other gene families can be discerned, providing a glimpse into their evolutionary history. Indeed, database homology searching indicates that VSIG4 is closely related to A33 antigen and junctional adhesion molecule A (JAM-A) but not to members of the B7 family. Phylogenetic analysis also supports this observation, showing that VSIG4, A33 antigen, and JAM-A group as a family that is distinct from the B7 family or complement receptor 1 (CR1) and CR2 family (Figure 1).

\section{CRIg: a complement receptor}

The complement system is activated by 3 different pathways: the classical, lectin, and alternative pathways. All 3 pathways share the common step of activating the central component, complement component 3 (C3). The binding of fragments derived from C3 activation and degradation to complement receptors results in the opsonization of pathogens, the attraction and activation of immune cells, and lysis of pathogens and infected cells. Four C3 fragment receptors were described a decade ago: CR1 (CD35), CR2 (CD21), CR3 (CD11b/CD18), and CR4 (CD11c/CD18) (9). CRIg/VSIG4 has now been added as the fifth member of this panel. 
The expression of VSIG4 on macrophages, a population of highly phagocytic cells, originally prompted Helmy et al. to test whether VSIG4 can directly interact with complement components on the cell surface (7). VSIG4 is able to bind IgMbound sheep erythrocytes in the presence of C3. More importantly, VSIG4 has been shown to be the receptor for $\mathrm{C} 3 \mathrm{~b}$ and $\mathrm{iC} 3 \mathrm{~b}$, 2 products of C3. During complement activation and particle opsonization, C3 is cleaved into multiple breakdown products. VSIG4 binds to plate-coated C3b and iC $3 \mathrm{~b}$ but not to other $\mathrm{C} 3$ products (such as $\mathrm{C} 3 \mathrm{a}$ and $\mathrm{C} 3 \mathrm{~d}$ ) or other complement components. Conversely, soluble C3b can also bind to plate-coated or cell-surface VSIG4. Taken together, these data demonstrate that both soluble and cell surfaceexpressed VSIG4 can bind soluble forms of $\mathrm{C} 3 \mathrm{~b}$ and $\mathrm{iC} 3 \mathrm{~b}$, as well as opsonized particles (7). Interestingly, VSIG4 may have a novel structure as a complement receptor, as it lacks C3b-and C4b-binding short consensus repeat sequences, which exist in CR1 and CR2 as well as the integrin-like domain present in CR3 and CR4.

The in vivo function of VSIG4 as a complement receptor has been analyzed using Vsig4-knockout mice (7). Liver macrophages (Kupffer cells) from Vsig4-deficient mice show a $60 \%$ reduction in their ability to bind C3-opsonized IgM-coated sheep erythrocytes. More importantly, Vsig4 is required for the clearance of $\mathrm{C} 3$-opsonized pathogens in vivo. Rapid capture of C3opsonized bacteria in the liver after introduction to the blood is crucial in preventing systemic bacteremia. After i.v. infection with either Listeria monocytogenes or Staphylococcus aureus, Vsig4-deficient Kupffer cells internalized fewer bacteria. As a result, significantly less live bacteria were recovered from the liver, and more were found in the blood, spleen, and lung in Vsig4-deficient mice. This defect in clearance of circulating bacteria by Vsig4-expressing liver Kupffer cells resulted in increased levels of serum cytokines, and these mice suffered increased mortality. Thus, through retention of C3-opsonized bacteria in the liver, Vsig4 not only helps to clear circulating bacteria but also to prevent infection of other organs, thereby promoting survival of the host. Given the importance of Vsig4 in bacterial clearance, it will be interesting to determine whether Vsig4-expressing macrophages play an important role in removing complement-tagged self antigens, such as apoptotic cells and cell remnants (10).

\section{VSIG4: a T cell inhibitor}

Given that VSIG4 is a distant relative rather than a bona fide homolog of the B7 family and has been demonstrated to be a complement receptor, the finding that it also has $T$ cell costimulatory activity is somewhat unexpected. Vogt et al. report that plate-coated VSIG4-Ig fusion protein can strongly inhibit proliferation and IL-2 production by $\mathrm{T}$ cells stimulated in vitro with anti-CD3 (5). Unlike some B7 family members that inhibit $\mathrm{T}$ cell function to a greater degree at low anti-CD3 antibody concentrations that induce weak TCR signals, VSIG4 strongly inhibits $\mathrm{CD}^{+}$and $\mathrm{CD}^{+} \mathrm{T}$ cells in the presence of both high and low concentrations of anti-CD3 antibodies and even in the presence of CD28 signal. Vogt et al. find that VSIG4-mediated inhibition of $\mathrm{T}$ cell proliferation is at least as potent as CD274- or PDCDILG2-mediated inhibition through PDCD1. One of main functional consequences of activation of the CD28 signal pathway is increased production of IL-2 by T cells. Interestingly, the inhibitory effect of VSIG4 on T cell proliferation can be abolished by the addition of IL-2 but not by the cross-linking of CD28. An important question remains to be answered regarding the biochemical signal that VISG4 triggers and its cross-regulation of TCR and CD28 signals.

Vogt et al. (5) go on to analyze the in vivo function of VSIG4 as a T cell inhibitor using a VSIG4-Ig fusion protein in vivo. Injection of soluble VSIG4-Ig protein decreases antigen-specific $\mathrm{CD}^{+} \mathrm{T}$ cells and IFN- $\gamma$ production in vivo and also reduces Th cell-dependent IgG responses. It is unclear how soluble VSIG4 works as a specific agonist but not as an antagonist in vivo, but it probably does so by cross-linking the VSIG4 receptor on T cells. Alternatively, the effect of VSIG4 in vivo could be attributed to its other role as a complement receptor, which in turn could be responsible for the reduction in immune responses. Given that human VSIG4 inhibits proliferation of both murine and human $\mathrm{T}$ cells (5), the negative regulatory function of this pathway is conserved between species. Therefore, Vogt et al. have identified a new macrophage ligand that may act as an attenuator of T cells.

\section{Dual functions reveal more questions than answers}

Thus, the papers published by Vogt et al. (5) and Helmy et al. (7) have identified the same molecule with 2 very different roles: as a $\mathrm{T}$ cell inhibitor and a complement receptor, respectively. These observations are surprising and raise some fundamental questions. First, how does VSIG4 work as a $\mathrm{T}$ cell inhibitor? At least 2 models could be proposed (Figure 1). One possibility is that VSIG4 binds to an as-yet-unidentified receptor expressed on $\mathrm{T}$ cells and induces a negative signal. No evidence, however, has been described supporting the existence of such a receptor $(5,7)$. Simple staining of $\mathrm{T}$ cells with recombinant VSIG4 protein (such as an Ig fusion or multimer) could provide valuable clues toward identifying this putative receptor and determining its mechanism of action. The other possibility is that VSIG4 inhibits T cell activation by acting as a complement receptor. Despite the fact that the expression of $\mathrm{C} 3$ receptors CR1 and CR3 on T cells was described many years ago (11-13), the function of these receptors on $\mathrm{T}$ cells is unclear. However, a recent study demonstrated that cross-linking CR1 and CR3 with antibodies profoundly inhibits $\mathrm{T}$ cell proliferation (14). In this context, it is noteworthy that the majority of in vivo $\mathrm{C} 3 \mathrm{~b}$ and $\mathrm{iC} 3 \mathrm{~b}$ molecules are present in multimeric form when deposited on particle surfaces (15, 16). Therefore, it is possible both VSIG4 on macrophages and CR1/CR3 on T cells bind the same multimeric $\mathrm{C} 3 \mathrm{~b}$ or $\mathrm{iC} 3 \mathrm{~b}$ molecules, which could in turn trigger a signal leading to $\mathrm{T}$ cell inhibition.

The second question is: what is the real physiological role of VSIG4? Kupffer cells from Vsig4-deficient mice are unable to efficiently clear C3-opsonized pathogens from the circulation, resulting in increased infection and mortality of the host. Unlike mice, however, human VSIG4 mRNA is expressed at very low levels in the liver (6, 7); further work is needed to determine the significance of VSIG4 as a liver complement receptor in humans. There is no information available about adaptive immune responses in Vsig4-deficient mice. It would be very informative to examine whether these mice have overreactive $\mathrm{T}$ cells and are predisposed to the development of autoimmunity due to the absence of Vsig4 inhibition. The role of Vsig4 as a T cell inhibitor could now be dissected by the use of Vsig-deficient mice.

The discovery of VSIG 4 as a T cell inhibitor as well as a complement receptor suggests that costimulation may be more complex than previously thought and provides an additional level of complexity. An appreciation of the possible dual roles of 
this molecule will have important implications for understanding diseases characterized by inappropriate $\mathrm{T}$ cell activation, including autoimmune diseases. To this end, however, we have a long way to go.

Address correspondence to: James P. Allison, Howard Hughes Medical Institute, Memorial Sloan-Kettering Cancer Center, 415 E. 68th Street, Box 470, New York, New York 10021, USA. Phone: (646) 888-2332; Fax: (646) 422-0470; E-mail: allisonj@mskcc.org.

1. Korman, A.J., Peggs, K.S., and Allison, J.P. 2006 Checkpoint blockade in cancer immunotherapy. Adv. Immunol. 90:297-339.

2. Peggs, K.S., Quezada, S.A., Korman, A.J., and Allison, J.P. 2006. Principles and use of anti-CTLA4 antibody in human cancer immunotherapy. Curr. Opin. Immunol. 18:206-213.

3. Okazaki, T., and Honjo, T. 2006. The PD-1-PD-L pathway in immunological tolerance. Trends Immunol.
27:195-201.

4. Barber, D.L., et al. 2006. Restoring function in exhausted CD8 T cells during chronic viral infection. Nature. 439:682-687.

5. Vogt, L., et al. 2006. VSIG4, a B7 family-related protein, is a negative regulator of $\mathrm{T}$ cell activation. J. Clin. Invest. 116:2817-2826. doi:10.1172/ JCI25673.

6. Langnaese, K., Colleaux, L., Kloos, D.U., Fontes, M., and Wieacker, P. 2000. Cloning of Z39Ig, a novel gene with immunoglobulin-like domains located on human chromosome X. Biochim. Biophys. Acta. 1492:522-525.

7. Helmy, K.Y., et al. 2006. CRIg: a macrophage complement receptor required for phagocytosis of circulating pathogens. Cell. 124:915-927.

8. Kim, J.K., et al. 2005. Characterization of monoclonal antibody specific to the Z39Ig protein, a member of immunoglobulin superfamily. Immunol. Lett. 99:153-161.

9. Holers, V.M., Kinoshita, T., and Molina, H. 1992. The evolution of mouse and human complement C3-binding proteins: divergence of form but conservation of function. Immunol. Today. 13:231-236.

10. Roozendaal, R., and Carroll, M.C. 2006. Emerging patterns in complement-mediated pathogen recognition. Cell. 125:29-32.

11. Gray, J.D., and Horwitz, D.A. 1988. Lymphocytes expressing type 3 complement receptors proliferate in response to interleukin 2 and are the precursors of lymphokine-activated killer cells. J. Clin. Invest. 81:1247-1254.

12. Claesson, M.H., and Miller, R.G. 1985. Functional heterogeneity in allospecific cytotoxic $\mathrm{T}$ lymphocyte clones. II. Development of syngeneic cytotoxicity in the absence of specific antigenic stimulation. J. Immunol. 134:684-690.

13. Yaskanin, D.D., Thompson, L.F., and Waxman, F.J. 1992. Distribution and quantitative expression of the complement receptor type 1 (CR1) on human peripheral blood T lymphocytes. Cell. Immunol. 142:159-176.

14. Wagner, C., et al. 2006. The complement receptor 1 , CR1 (CD35), mediates inhibitory signals in human T-lymphocytes. Mol. Immunol. 43:643-651.

15. Hong, K., et al. 1991. Reconstitution of C5 convertase of the alternative complement pathway with isolated C3b dimer and factors B and D. J. Immunol. 146:1868-1873

16. Brown, E.J. 1991. Complement receptors and phagocytosis. Curr. Opin. Immunol. 3:76-82.

\title{
Children are not little adults: just ask their hematopoietic stem cells
}

\author{
David A. Williams, ${ }^{1}$ Haiming $X u,{ }^{1}$ and Jose A. Cancelas ${ }^{1,2}$
}

1Division of Experimental Hematology, Cincinnati Children's Research Foundation and Cincinnati Children's Hospital Medical Center, Cincinnati, Ohio, USA. ${ }^{2}$ Hoxworth Blood Center, University of Cincinnati Academic Health Center, Cincinnati, Ohio, USA.

HSCs differ during ontogeny in some important parameters, including anatomic site of residence and cell cycling characteristics. In this issue of the JCI, Bowie et al. show that postnatal HSCs as well as fetal liver HSCs in mice are active in the cell cycle at much higher rates than that of adult HSCs; however, this increased frequency of cycling abruptly ceases 4 weeks after birth (see the related article beginning on page 2808). The cycling postnatal HSCs expressed high levels of CXC chemokine ligand 12 (CXCL12, also known as stromal cell-derived factor 1 [SDF-1]), a chemokine previously implicated in stem cell trafficking to the marrow cavity and shown to be expressed by cells within the hematopoietic microenvironment. These cells also possessed an engraftment defect impeding reconstitution in irradiated recipient mice, which was reversible by pretransplant administration of antagonists of the CXCL12 receptor, CXCR4. Such agents are currently clinically available, suggesting that this approach could be used to improve stem cell transplantation and engraftment.

HSCs, similar to all stem cells, have 2 defining properties: the capacity for multilineage differentiation and the capacity to generate daughter stem cells with identical proliferative/differentiation capacity through a

Nonstandard abbreviations used: CXCL12, CXC chemokine ligand 12; CXCR4, CXC chemokine receptor 4.

Conflict of interest: The authors have declared that no conflict of interest exists.

Citation for this article: J. Clin. Invest. 116:2593-2596 (2006). doi:10.1172/JCI30083. self-renewal process. Thus, HSCs comprise a compartment of undifferentiated stem cells while continuously providing more differentiated progenitor cells that are the more proximate source of all circulating blood cells. Adult HSCs are largely quiescent. It is estimated that approximately $95 \%$ of HSCs responsible for engraftment in stem cell transplantation assays are in the $\mathrm{G}_{0}$ phase of the cell cycle $(1,2)$. These stem cells show a slow rate of turnover, while their progenitor progeny, the majority of which are actively cycling, are responsible for the vast majority of the cell amplification that is necessary for the production of billions of blood cells each day.

\section{Hematopoiesis changes during ontogeny}

The stem cell compartment expands enormously during fetal development and after HSC transplantation as well as in times of hematopoietic stress. This expansion is associated with a much higher frequency of cycling HSCs, especially during embryonic and fetal stages of development. The anatomic site of hematopoiesis is also distinct in the fetus versus the adult, with the major site of blood formation occurring successively in the yolk sac, the aorta-gonadmesonephros, and the fetal liver during embryonic development (1-3), while it is restricted to the bone marrow cavity in adults (and the spleen in mice) (4).

Along with these changes in anatomic location of blood formation, there are important differences in other aspects of hematopoiesis during development. For instance, embryonic (yolk sac-derived) red blood cells are nucleated, while fetal 